\title{
WEIGHTED INF-SUP CONDITION AND POINTWISE ERROR ESTIMATES FOR THE STOKES PROBLEM
}

\author{
RICARDO G. DURAN AND RICARDO H. NOCHETTO
}

\begin{abstract}
Convergence of mixed finite element approximations to the Stokes problem in the primitive variables is examined in maximum norm. Quasioptimal pointwise error estimates are derived for discrete spaces satisfying a weighted inf-sup condition similar to the Babuška -Brezzi condition. The usual techniques employed to prove the inf-sup condition in energy norm can be easily extended to the present situation, thus providing several examples to our abstract framework. The popular Taylor-Hood finite element is the most relevant one.
\end{abstract}

\section{INTRODUCTION}

The Stokes problem, which describes the flow of a viscous incompressible fluid, consists of finding $\underset{\sim}{u}$ and $p$ so that

$$
\begin{aligned}
-\Delta \underset{\sim}{u}+\nabla p=\underset{\sim}{f} & \text { in } \Omega, \\
\operatorname{div} \underset{\sim}{u}=0 & \text { in } \Omega, \\
\underset{\sim}{u}=\underset{\sim}{\sim} & \text { on } \partial \Omega,
\end{aligned}
$$

where $\underset{\sim}{u}$ indicates the velocity and $p$ the pressure, $\underset{\sim}{f}$ denotes a given external force and $\Omega$ is a bounded domain contained in $\mathbb{R}^{2}$. This is the formulation in the primitive variables $\underset{\sim}{u}$ and $p$, also called velocity-pressure formulation. A weak form of (1.1) suitable for finite element approximations is the problem of seeking $\underset{\sim}{u} \in \underset{\sim}{\mathbf{V}}:=\left[H_{0}^{1}(\Omega)\right]^{2}$ and $p \in \mathbf{P}:=L_{0}^{2}(\Omega)$ such that

$$
\begin{aligned}
\langle\nabla \underset{\sim}{u}, \nabla \underset{\sim}{v}\rangle-\langle\operatorname{div} \underset{\sim}{v}, p\rangle & =\langle\underset{\sim}{f}, \underset{\sim}{v}\rangle, & & \forall \underset{\sim}{v} \in \underset{\sim}{\mathbf{V}}, \\
\langle\operatorname{div} \underset{\sim}{u}, q\rangle & =0, & & \forall q \in \mathbf{P},
\end{aligned}
$$

where $\langle\cdot, \cdot\rangle$ denotes the inner product in $L^{2}(\Omega)$, and $L_{0}^{2}(\Omega)$ is the space of $L^{2}$-functions having mean value zero.

Received August 18, 1988; revised April 13, 1989.

1980 Mathematics Subject Classification (1985 Revision). Primary 65N15, 65N30, 76D07; Secondary 35B45, 35J50.

Key words and phrases. Stokes problem, mixed finite elements, pointwise error estimates.

This work was partially supported by NSF Grant DMS-8805218 and by the Institute for Mathematics and its Applications with funds provided by the NSF. 
We assume that $\Omega$ is a convex polygon and $\left\{\mathscr{T}_{h}\right\}_{h}$ is a regular and quasiuniform family of decompositions of $\Omega$ [15, pp. 132, 140]; $h$ stands for the mesh size. Let $\mathbf{V}_{h} \subset \underset{\sim}{\mathbf{V}}$ and $\mathbf{P}_{h} \subset \mathbf{P}$ denote finite element spaces associated with $\mathscr{T}_{h}$. The discrete problem then reads as follows: find $\underset{\sim}{u_{h}} \in \underset{\sim}{\mathbf{V}_{h}}$ and $p_{h} \in \mathbf{P}_{h}$ such that

$$
\begin{array}{rlrl}
\left\langle\nabla \underset{\sim}{u_{h}}, \nabla \underset{\sim}{v}\right\rangle-\left\langle\operatorname{div} \underset{\sim}{v}, p_{h}\right\rangle & =\langle\underset{\sim}{f}, \underset{\sim}{v}\rangle, & \forall \underset{\sim}{v} \in{\underset{\sim}{\mathbf{V}}}_{h}, \\
\left\langle\operatorname{div} \underset{\sim}{u_{h}}, q\right\rangle=0, & \forall q \in \mathbf{P}_{h} .
\end{array}
$$

The discrete spaces ${\underset{\sim}{h}}_{h}$ and $\mathbf{P}_{h}$ must satisfy a compatibility condition for (1.3) to be stable. This constraint is expressed by the celebrated discrete inf-sup condition

$$
\sup _{\substack{v \in \mathbf{V}_{h} \\ \sim}} \frac{\langle\operatorname{div} \underset{\sim}{v}, q\rangle}{\|\nabla \underset{\sim}{v}\|_{L^{2}(\Omega)}} \geq \beta\|q\|_{L^{2}(\Omega)}, \quad \forall q \in \mathbf{P}_{h}
$$

where $\beta$ is independent of $h[3,7,15]$. This leads to optimal-order energy error estimates as well $[3,7,15]$.

The continuous inf-sup condition is equivalent to having a bounded right inverse for the divergence operator subject to homogeneous Dirichlet boundary conditions; this issue is discussed in [2] for polygonal domains. Here we show that there exists a right inverse for the divergence operator which is almost bounded in weighted Sobolev spaces; this is the only two-dimensional result of this paper. This crucial property enables us to introduce a proper notion of discrete weighted inf-sup condition and, in addition, to demonstrate that usual techniques employed to prove (1.4) can be generalized so as to lead to our weighted inf-sup condition. Several examples such as the popular Taylor-Hood finite element illustrate the theory.

We then derive quasi-optimal maximum norm error estimates for spaces satisfying the weighted inf-sup condition; the error analysis is $n$-dimensional. The main tool is, as usual, the method of weighted Sobolev norms introduced by Natterer [17] and Nitsche [18]. Our present results contain those in [11], which rely on locally constructing the so-called Fortin's operator.

An outline of the paper is as follows. In $\S 2$ we prove several a priori estimates in weighted norms. In $\S 3$ we introduce the weighted inf-sup condition and extend standard techniques in energy norm to this new situation; several examples illustrate our results. We conclude in $\S 4$ with the error analysis in maximum norm.

\section{Weighted A PRIORI ESTIMATES}

We start this section by recalling the definition of the usual weight function along with some of its fundamental properties.

The weight function $\sigma$ is defined by

$$
\sigma(x):=\left(\left|x-x_{0}\right|^{2}+\theta^{2}\right)^{1 / 2} \text { for } x, x_{0} \in \Omega,
$$


where $\theta \gg h$ is a small parameter to be determined later on. It is well known that $\sigma$ satisfies the following properties $[8,18]$ :

$$
\begin{gathered}
\max _{T \in J_{h}}\left[\max _{x \in T} \sigma(x) / \min _{x \in T} \sigma(x)\right] \leq C, \\
\left|D^{j} \sigma^{\alpha}(x)\right| \leq C(j, \alpha) \sigma^{\alpha-j}(x), \quad \forall x \in \Omega,
\end{gathered}
$$

where $\alpha \in \mathbb{R}$ and $D^{j} f$ denotes the tensor of $j$ th derivatives of $f$. Hereafter, the letter $C$ will indicate a positive constant independent of $h$ and $x_{0}$, but not necessarily the same at each occurrence. Moreover, a simple calculation shows that

$$
\int_{\Omega} \sigma(x)^{-(2+\alpha)} d x \leq \begin{cases}C \theta^{-\alpha}, & \alpha>0, \\ C|\log \theta|, & \alpha=0,\end{cases}
$$

for $\theta$ sufficiently small. For $\alpha \in \mathbb{R}$ and $j \in \mathbb{N}$, the weighted Sobolev seminorms are defined by

$$
\left\|D^{j} q\right\|_{\sigma^{\prime \prime}}^{2}:=\sum_{|\beta|=j} \int_{\Omega}\left\|\partial^{\beta} q\right\|^{2} \sigma^{\alpha}, \quad \forall q \in H^{j}(\Omega) ;
$$

the same notation will be used for vector-valued functions.

We assume that for some $k \geq 1$,

$$
\left.\mathbf{V}_{h}\right|_{T} \supset\left[P_{k}(T)\right]^{2},\left.\quad \mathbf{P}_{h}\right|_{T} \supset P_{k-1}(T), \quad \forall T \in \mathscr{T}_{h},
$$

where $P_{k}(T)$ stands for the space of polynomials of total degree not greater than $k$ restricted to $T$. Given $q \in L^{2}(\Omega)$ (or $\underset{\sim}{v} \underset{\sim}{\mathbf{V}}$ ), the symbol $\hat{q}=I_{h} q \in \mathbf{P}_{h} \oplus \mathbb{R}$ (or $\underset{\sim}{\hat{v}}=I_{h} \underset{\sim}{v} \in{\underset{\sim}{h}}_{h}$ ) indicates either its local average interpolant [15, p. 109] for continuous elements or its local $L^{2}$-projection for discontinuous ones. In view of its local character, $I_{h}$ is an optimal-order interpolation operator in $L^{p}(\Omega)$ $(1 \leq p \leq \infty)$ as well as in weighted Sobolev norms. Moreover, the following superapproximation properties hold:

$$
\begin{aligned}
\left\|\sigma^{-2} q-I_{h}\left(\sigma^{-2} q\right)\right\|_{\sigma^{2}} & \leq C \frac{h}{\theta}\|q\|_{\sigma^{-2}}, \\
\left\|\nabla\left[\sigma^{-2} q-I_{h}\left(\sigma^{-2} q\right)\right]\right\|_{\sigma^{2}} & \leq C \frac{h}{\theta}\left(\|q\|_{\sigma^{-4}}+\|\nabla q\|_{\sigma^{-2}}\right),
\end{aligned}
$$

for all $q \in \mathbf{P}_{h} \oplus \mathbb{R}$; similar bounds are valid for all $\underset{\sim}{v} \in \underset{\sim}{\mathbf{V}_{h}}$. These estimates come from applying the Bramble-Hilbert lemma.

We now turn our attention to a priori estimates in weighted norms. Our first result is restricted to two dimensions and will play a relevant role afterwards.

Lemma 2.1. $\|\varphi\|_{\sigma^{-2}} \leq C|\log \theta|^{1 / 2}\|\varphi\|_{H^{1}(\Omega)}, \quad \forall \varphi \in H^{1}(\Omega)$.

Proof. By the Sobolev imbedding theorem in two dimensions [19], we have

$$
\|\varphi\|_{L^{s}(\Omega)} \leq C s^{1 / 2}\|\varphi\|_{H^{1}(\Omega)}, \quad \forall 2<s<\infty .
$$


Making now use of Hölder's inequality together with (2.4) yields

$$
\|\varphi\|_{\sigma^{-2}} \leq\left(\int_{\Omega}|\varphi|^{2 p}\right)^{1 / 2 p}\left(\int_{\Omega} \sigma^{-2 p /(p-1)}\right)^{(p-1) / 2 p} \leq C p^{1 / 2} \theta^{-1 / p}\|\varphi\|_{H^{\mathrm{i}}(\Omega)} .
$$

The desired result then follows from taking $p=|\log \theta|$.

To proceed further, we need some well-known energy a priori estimates for the Stokes problem. Let $\underset{\sim}{b} \in\left[H^{-1}(\Omega)\right]^{2}$ and $g \in L_{0}^{2}(\Omega)$. Then there exists a unique solution $(\underset{\sim}{v}, q) \in \underset{\sim}{\tilde{\mathbf{V}}} \times \mathbf{P}$ to the generalized Stokes problem $[15,21]$

$$
\begin{aligned}
-\Delta v_{\sim}^{v}+\nabla q=\underset{\sim}{b} & \text { in } \Omega, \\
\operatorname{div} v=g & \text { in } \Omega,
\end{aligned}
$$

which satisfies

$$
\|\underset{\sim}{v}\|_{H^{1}(\Omega)}+\|q\|_{L^{2}(\Omega)} \leq C\left(\|\underset{\sim}{b}\|_{H^{-1}(\Omega)}+\|g\|_{L^{2}(\Omega)}\right) .
$$

In addition, if $\underset{\sim}{b} \in\left[L^{2}(\Omega)\right]^{2}$ and $g \in H_{0}^{1}(\Omega)$, then [16]

$$
\|\underset{\sim}{v}\|_{H^{2}(\Omega)}+\|q\|_{H^{1}(\Omega)} \leq C\left(\|\underset{\sim}{b}\|_{L^{2}(\Omega)}+\|\nabla g\|_{L^{2}(\Omega)}\right) \text {. }
$$

We are now in a position to prove a crucial result. It roughly says that the divergence operator possesses a right inverse which is almost bounded (uniformly in $h$ ) in weighted Sobolev spaces.

Lemma 2.2. Given $g \in L^{2}(\Omega)$, set $m:=|\Omega|^{-1} \int_{\Omega} g$. Then there exists $\underset{\sim}{v} \in \underset{\sim}{\mathbf{V}}$ such that

$$
\operatorname{div} \underset{\sim}{v}=g-m \text { in } \Omega
$$

and, moreover,

$$
\|\nabla \underset{\sim}{v}\|_{\sigma^{2}} \leq C|\log \theta|^{1 / 2}\|g\|_{\sigma^{2}} .
$$

Proof. In order to construct $\underset{\sim}{v} \in \underset{\sim}{\mathbf{V}}$, we resort to the generalized Stokes problem.

Let $\underset{\sim}{v} \in \mathbf{\sim}$ be the solution to

$$
\begin{aligned}
-\Delta \underset{\sim}{v}+\nabla q & =\underset{\sim}{0}, \\
\operatorname{div} \underset{\sim}{v} & =g-m .
\end{aligned}
$$

Here, $q$ denotes a function in $L^{2}(\Omega)$ having a mean value to be determined later on. To prove (2.10), it suffices to deal with the components $\mu_{j}=x_{j}-x_{j}^{0}$ $(j=1,2)$ of $x-x_{0}$ rather than $\sigma[8$, p. 148]. Since no confusion is possible, we remove the subscript $j$. We now observe that $(\mu \underset{\sim}{v}, \mu q)$ satisfies the pair of equations

$$
\begin{aligned}
-\Delta(\underset{\sim}{\mu v})+\nabla(\mu q) & =-2 \nabla \mu \cdot \nabla \underset{\sim}{v}+q \nabla \mu, \\
\operatorname{div}(\mu \underset{\sim}{v}) & =\mu(g-m)+\underset{\sim}{v} \cdot \nabla \mu .
\end{aligned}
$$


Hence (2.7), in conjunction with the fact that $\mu$ is linear, yields

$$
\|\nabla(\mu \underset{\sim}{v})\|_{L^{2}(\Omega)} \leq C\left(\|\underset{\sim}{v}\|_{L^{2}(\Omega)}+\|q\|_{H^{-1}(\Omega)}+\|\mu(g-m)\|_{L^{2}(\Omega)}\right) .
$$

Our next task is to evaluate the right-hand side of this expression. To bound $\|q\|_{H^{-1}(\Omega)}$, we need to introduce an auxiliary function $\eta \in C_{0}^{\infty}(\Omega)$ so that $\int_{\Omega} \eta=1$. Since $q$ is defined up to a constant, we can choose it so that $\int_{\Omega} q \eta=$ 0 . Given $\varphi \in H_{0}^{1}(\Omega)$, let $(\underset{\sim}{\psi}, \lambda) \in \underset{\sim}{\mathbf{V}} \times \mathbf{P}$ be the solution to the auxiliary problem

$$
\begin{aligned}
&-\Delta \underset{\sim}{\psi}+\nabla \lambda=\underset{\sim}{0}, \\
& \operatorname{div} \underset{\sim}{\psi}=\varphi-\eta r,
\end{aligned}
$$

where $r:=\int_{\Omega} \varphi$. Since the compatibility condition $\int_{\Omega} \varphi-\eta r=0$ holds, the previous problem admits a unique solution. Thus

$$
\langle q, \varphi\rangle=\langle q, \varphi-\eta r\rangle=\langle q, \operatorname{div} \underset{\sim}{\psi}\rangle=\langle\underset{\sim}{v}, \nabla \underset{\sim}{\psi}\rangle=-\langle\underset{\sim}{v}, \Delta \underset{\sim}{\psi}\rangle,
$$

because $\varphi-\eta r \in H_{0}^{1}(\Omega)$ yields $\underset{\sim}{\psi} \in\left[H^{2}(\Omega)\right]^{2}$. Moreover, from (2.8) we deduce that

$$
|\langle q, \varphi\rangle| \leq C\|\underset{\sim}{v}\|_{L^{2}(\Omega)}\|\varphi-\eta r\|_{H_{0}^{1}(\Omega)} \leq C\|\underset{\sim}{v}\|_{L^{2}(\Omega)}\|\varphi\|_{H_{0}^{1}(\Omega)} ;
$$

therefore,

$$
\|q\|_{H^{-1}(\Omega)} \leq C\|\underset{\sim}{v}\|_{L^{2}(\Omega)} .
$$

To estimate $\|\underset{\sim}{v}\|_{L^{2}(\Omega)}$, we employ a duality argument. Let $(\underset{\sim}{\psi}, \lambda) \underset{\sim}{\mathbf{V}} \times \mathbf{P}$ be the solution to the auxiliary problem

$$
\begin{aligned}
&-\Delta \underset{\sim}{\psi}+\nabla \lambda=\underset{\sim}{v}, \\
& \operatorname{div} \underset{\sim}{\psi}=0 .
\end{aligned}
$$

In view of $(2.11)$ we can write

$$
\begin{aligned}
\|\underset{\sim}{v}\|_{L^{2}(\Omega)}^{2} & =\langle\underset{\sim}{\sim}, \nabla \underset{\sim}{\sim}\rangle-\langle\operatorname{div} \underset{\sim}{\sim}, \lambda\rangle=\langle q, \operatorname{div} \underset{\sim}{\sim}\rangle-\langle g-m, \lambda\rangle \\
& =-\langle g, \lambda\rangle \leq\|g\|_{\sigma^{2}}\|\lambda\|_{\sigma^{-2}} .
\end{aligned}
$$

Using Lemma 2.1, in conjunction with $(2.8)$, yields

$$
\|v\|_{L^{2}(\Omega)} \leq C|\log \theta|^{1 / 2}\|g\|_{\sigma^{2}} .
$$

It only remains to estimate the rightmost term in (2.12). It follows that

$$
\begin{aligned}
\|\mu(g-m)\|_{L^{2}(\Omega)}^{2} & \leq \int_{\Omega} \sigma^{2}|g-m|^{2} \leq C\left(\int_{\Omega} \sigma^{2}|g|^{2}+m^{2} \int_{\Omega} \sigma^{2}\right) \\
& \leq C|\log \theta|\|g\|_{\sigma^{2}}^{2},
\end{aligned}
$$

where we have used (2.4) and the Cauchy-Schwarz inequality to bound $\mathrm{m}^{2}$. Replacing the above estimates in (2.12), we find that

$$
\|\nabla(\mu \underset{\sim}{v})\|_{L^{2}(\Omega)} \leq C|\log \theta|^{1 / 2}\|g\|_{\sigma^{2}}
$$


This, combined with the equality

$$
\|\nabla \underset{\sim}{v}\|_{\sigma^{2}}^{2}=\theta^{2}\|\nabla \underset{\sim}{v}\|_{L^{2}(\Omega)}^{2}+\sum_{j=1}^{2}\left\|\mu_{j} \nabla \underset{\sim}{v}\right\|_{L^{2}(\Omega)}^{2}
$$

and (2.7), implies the asserted estimate.

Remark 2.1. The previous argument is essentially two-dimensional. The natural $n$-dimensional analogue of (2.10) would be

$$
\|\nabla \underset{\sim}{v}\|_{\sigma^{n}} \leq C|\log \theta|^{1 / 2}\|g\|_{\sigma^{n}} .
$$

If this estimate were available, then the remainder of this paper could be easily extended to higher dimensions.

We conclude this section with an a priori estimate which extends (2.8) to weighted Sobolev spaces.

Lemma 2.3. Let $\underset{\sim}{b} \in\left[L^{2}(\Omega)\right]^{2}, g \in H_{0}^{1}(\Omega)$ and $\phi \in C_{0}^{\infty}(\Omega)$ be given, with $\int_{\Omega} \phi=1$. Let $(\underset{\sim}{v}, q) \in \underset{\sim}{\mathbf{V}} \times \mathbf{P}$ be the solution to

$$
\begin{aligned}
-\Delta \underset{\sim}{v}+\nabla q & =\underset{\sim}{b} & & \text { in } \Omega, \\
\operatorname{div} v & =g-m \phi & & \text { in } \Omega,
\end{aligned}
$$

where $m:=\int_{\Omega} g$. Then there exists a constant $C>0$ such that

$$
\left\|D^{2} \underset{\sim}{v}\right\|_{\sigma^{2}}+\|\nabla q\|_{\sigma^{2}} \leq C\left(|\log \theta|^{1 / 2}\|\underset{\sim}{b}\|_{\sigma^{2}}+\|\nabla g\|_{\sigma^{2}}+\|g\|_{L^{2}(\Omega)}\right) .
$$

Proof. As in the previous lemma, we deal with the components $\mu_{j}$ of $x-x_{0}$ instead of $\sigma$, and we remove the subscript $j$. A simple calculation leads to

$$
\begin{aligned}
-\Delta(\mu \underset{\sim}{v})+\nabla(\mu q) & =\mu \underset{\sim}{b}-2 \nabla \mu \cdot \nabla \underset{\sim}{v}+q \nabla \mu, \\
\operatorname{div}(\mu \underset{\sim}{v}) & =\mu(g-m \phi)+\nabla \mu \cdot \underset{\sim}{v} .
\end{aligned}
$$

Using the fact that $\mu$ is linear, and (2.8), we easily obtain

$$
\begin{aligned}
\left\|D^{2}(\mu \underset{\sim}{v})\right\|_{L^{2}(\Omega)}+\|\nabla(\mu q)\|_{L^{2}(\Omega)} \leq & C\left(\left\|\mu \underset{\sim}{b \|_{L^{2}(\Omega)}}+\right\| g-m \phi \|_{L^{2}(\Omega)}\right. \\
& \left.+\|\mu \nabla(g-m \phi)\|_{L^{2}(\Omega)}+\|\nabla \underset{\sim}{v}\|_{L^{2}(\Omega)}+\|q\|_{L^{2}(\Omega)}\right) .
\end{aligned}
$$

We start examining the last two terms. In view of (2.7), we have to bound $\|\underset{\sim}{b}\|_{H^{-1}(\Omega)}$ and $\|g-m \phi\|_{L^{2}(\Omega)}$. Given $\underset{\sim}{\varphi} \in \underset{\sim}{\mathbf{V}}$, we can make use of Lemma 2.1 to arrive at

$$
|\langle\underset{\sim}{b}, \underset{\sim}{\varphi}\rangle| \leq\|\underset{\sim}{b}\|_{\sigma^{2}}\|\underset{\sim}{\varphi}\|_{\sigma^{-2}} \leq C|\log \theta|^{1 / 2}\|\underset{\sim}{b}\|_{\sigma^{2}}\|\underset{\sim}{\varphi}\|_{H^{1}(\Omega)}
$$

hence,

$$
\|\underset{\sim}{b}\|_{H^{-1}(\Omega)} \leq C|\log \theta|^{1 / 2}\|\underset{\sim}{b}\|_{\sigma^{2}} .
$$

At the same time, as $m=\int_{\Omega} g \leq C\|g\|_{L^{2}(\Omega)}$, we also have $\|g-m \phi\|_{L^{2}(\Omega)} \leq$ $C\|g\|_{L^{2}(\Omega)}$. We finally argue as in the end of the proof to Lemma 2.2 to obtain the desired result. 
Remark 2.2. It is not too difficult to generalize Lemma 2.3 to higher dimensions. In fact, assuming that $\Omega$ is sufficiently smooth, thus having precise pointwise bounds for the Green's function of the Stokes problem, one might proceed as in [14, Lemma 3.2] to end up with

$$
\left\|D^{2} \underset{\sim}{v}\right\|_{\sigma^{n}}+\|\nabla q\|_{\sigma^{n}} \leq C \frac{|\log \theta|^{1 / 2}}{\theta}\left(\|\underset{\sim}{b}\|_{\sigma^{n+2}}+\|\nabla g\|_{\sigma^{n+2}}+\|g\|_{\sigma^{n}}\right) .
$$

It turns out that this estimate suffices for later purposes.

\section{WEIGHTED INF-SUP CONDITION}

In this section we introduce a proper notion of discrete weighted inf-sup condition, which is based on a crucial property fulfilled by the divergence operator. This will be our main hypothesis in the subsequent error analysis. We also demonstrate that standard techniques used to prove the inf-sup condition in energy norm can be suitably extended to the present situation, thus providing several examples.

Lemma 3.1. There exists a constant $C>0$ such that

$$
\sup _{v \in \underset{\sim}{\mathbf{V}}} \frac{\langle\operatorname{div} \underset{\sim}{v}, q\rangle}{\|\nabla \underset{\sim}{v}\|_{\sigma^{2}}} \geq C|\log \theta|^{-1 / 2}\|q\|_{\sigma^{-2}}, \quad \forall q \in \mathbf{P} .
$$

Proof. Given $q \in \mathbf{P}$, set $g:=\sigma^{-2} q$ and let $\underset{\sim}{v} \in \underset{\sim}{\mathbf{V}}$ indicate the function in (2.9). Hence,

$$
\|\nabla \underset{\sim}{v}\|_{\sigma^{2}} \leq C|\log \theta|^{1 / 2}\left\|\sigma^{-2} q\right\|_{\sigma^{2}}=C|\log \theta|^{1 / 2}\|q\|_{\sigma^{-2}}
$$

which, in turn, implies (3.1).

Remark 3.1. It would be of interest to know whether or not the inf-sup constant $\beta_{\theta}=C|\log \theta|^{-1 / 2}$ is optimal.

In view of (3.1) we say that a pair $\left(\mathbf{V}_{h}, \mathbf{P}_{h}\right)$ satisfies the discrete weighted inf-sup condition if there exists $C>0$ independent of $h$ such that

$$
\sup _{\underset{\sim}{v \in \mathbf{V}_{h}}} \frac{\langle\operatorname{div} \underset{\sim}{v}, q\rangle}{\|\nabla \underset{\sim}{v}\|_{\sigma^{2}}} \geq C|\log \theta|^{-1 / 2}\|q\|_{\sigma^{-2}}, \quad \forall q \in \mathbf{P}_{h} .
$$

The generalization to higher dimensions consists of simply replacing $\sigma^{2}$ and $\sigma^{-2}$ by $\sigma^{n}$ and $\sigma^{-n}$, respectively.

Remark 3.2. The subsequent error analysis relies mainly on the structure of (3.2) rather than on the particular expression of $\beta_{\theta}$.

Our next goal is to develop two techniques for the effective verification of (3.2) and to illustrate their application to well-known finite element spaces. The first one is based on the fact that condition (1.4) is equivalent to the existence of a global operator $\Pi_{h}: \underset{\sim}{\mathbf{V}} \rightarrow \underset{\sim}{\mathbf{V}_{h}}$ so that [12]

$$
\begin{gathered}
\left\langle\operatorname{div}\left(\underset{\sim}{v}-\Pi_{h} \underset{\sim}{v}\right), q\right\rangle=0, \quad \forall q \in \mathbf{P}_{h}, \\
\left\|\nabla \Pi_{h} \underset{\sim}{v \|_{L^{2}(\Omega)}} \leq C\right\| \nabla \underset{\sim}{v} \|_{L^{2}(\Omega)} .
\end{gathered}
$$


In many practical situations $\Pi_{h}$ can be constructed locally. In this case, quasioptimal pointwise error estimates have been derived in two dimensions using a different approach; see [11] where also several examples were reported. The following lemma, which resembles the so-called Fortin's trick [13], shows that (3.2) is valid as soon as we have

$$
\left\|\nabla \Pi_{h} \underset{\sim}{v}\right\|_{\sigma^{2}} \leq C\|\nabla \underset{\sim}{v}\|_{\sigma^{2}}, \quad \forall \underset{\sim}{v} \in \underset{\sim}{\mathbf{V}} .
$$

This property is an easy consequence of (2.2) provided $\Pi_{h}$ is a local operator or, equivalently, whenever (3.4) is valid locally. Note that $\Pi_{h}$ is not required to be defined elementwise for (3.5) to hold. Indeed, the definition of $\Pi_{h}$ on one element may also involve the adjacent ones, as customary when dealing with the local average interpolant [15, p. 109;1].

Lemma 3.2. Let $\Pi_{h}$ satisfy (3.3) and (3.5). Then (3.2) holds.

Proof. Let $q \in \mathbf{P}_{h} \subset \mathbf{P}$. By virtue of Lemma 3.1, there exists $\underset{\sim}{v} \in \underset{\sim}{\mathbf{V}}$ such that

$$
\frac{\langle\operatorname{div} \underset{\sim}{v}, q\rangle}{\|\nabla \underset{\sim}{v}\|_{\sigma^{2}}} \geq C|\log \theta|^{-1 / 2}\|q\|_{\sigma^{-2}} .
$$

Next, set $\underset{\sim}{v_{h}}:=\Pi_{h} \underset{\sim}{v} \in \mathbf{V}_{h}$ and use (3.3) and (3.5) to get (3.2).

We now consider some examples for which Lemma 3.2 applies. Let $\mathscr{T}_{h}$ be made of triangles $T$ and let $\lambda_{1}, \lambda_{2}, \lambda_{3}$ denote the barycentric coordinates of $T$.

Example 3.1. Mini Element. It was introduced by Arnold, Brezzi and Fortin [1] as a remedy for the unstable $P_{1}-P_{1}$ element. The discrete spaces are defined by

$$
\left.{\underset{\sim}{h}}_{T}\right|_{T}:=\left[P_{1}(T) \oplus \lambda_{1} \lambda_{2} \lambda_{3} P_{0}(T)\right]^{2},\left.\quad \mathbf{P}_{h}\right|_{T}:=P_{1}(T), \quad \forall T \in \mathscr{T}_{h},
$$

and $\mathbf{P}_{h} \subset C^{0}(\Omega)$; thus $k=1$. The local operator $\Pi_{h}$ was explicitly built in [1] as a means to demonstrate stability (see also [15, p. 175]).

Example 3.2. Bernardi-Raugel Element. The discrete spaces are defined by [5; 15, p. 134]

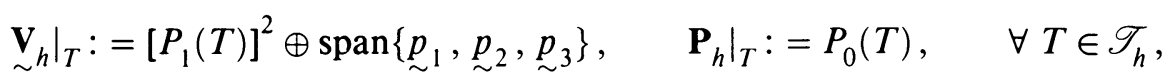

where $\underset{\sim}{p}:=\lambda_{2} \lambda_{3}{\underset{\sim}{1}}_{1}, \underset{\sim}{p}:=\lambda_{1} \lambda_{3} \nu_{\sim}$ and $\underset{\sim}{p_{3}}:=\lambda_{1} \lambda_{2}{\underset{\sim}{3}}_{3}, \nu_{\sim}{ }_{i}$ being the unit vector normal to the opposite side to vertex $i$; thus $k=1$. This element may be viewed as a simplification of the classical $P_{2}-P_{0}$ element which turns out to fit in our theory as well.

In many circumstances the operator $\Pi_{h}$ is not known to be local, as happens for the Taylor-Hood finite element [15, 20]; so (3.5) might fail to hold. Our next goal is thus to generalize a method due to Boland and Nicolaides [6], which reduces the proof of (1.4) to the verification of a local inf-sup condition. To this end, we need some extra notation. Let $\mathbf{Q}_{h} \subset L^{2}(\Omega)$ be so that $\mathbf{P}_{h}=\mathbf{Q}_{h} \cap \mathbf{P}$, 
and assume that $\mathbf{Q}_{h}$ contains the piecewise constant functions. Let $\Omega$ be decomposed into a finite number of disjoint, connected and open macroelements $\Omega_{r}$ which, in turn, are unions of a number of finite elements bounded uniformly in $h$; hence $\operatorname{diam}\left(\Omega_{r}\right) \leq C h$ for all $1 \leq r \leq R$. Set

$$
\begin{aligned}
& \underset{\sim}{\mathbf{V}_{r}}:=\left\{\underset{\sim}{v} \in \underset{\sim}{\mathbf{V}_{h}}: \underset{\sim}{v}=\underset{\sim}{0} \text { in } \Omega \backslash \Omega_{r}\right\}, \\
& \mathbf{Q}_{r}:=\left\{\left.q\right|_{\Omega_{r}}: q \in \mathbf{Q}_{h}\right\}, \quad \mathbf{P}_{r}:=\mathbf{Q}_{r} \cap L_{0}^{2}\left(\boldsymbol{\Omega}_{r}\right), \\
& \overline{\mathbf{P}}_{h}:=\left\{q \in \mathbf{P}:\left.q\right|_{\Omega_{r}} \text { is constant }, \quad 1 \leq r \leq R\right\} .
\end{aligned}
$$

Lemma 3.3. Let the pairs $\left.\underset{\sim}{\mathbf{V}_{r}}, \mathbf{P}_{r}\right)$ satisfy the local inf-sup condition

$$
\sup _{\substack{v \in \mathbf{v}_{r} \\ \sim}} \frac{\langle\operatorname{div} \underset{\sim}{v}, q\rangle}{\|\underset{\sim}{v}\|_{L^{2}\left(\Omega_{r}\right)}} \geq \gamma\|q\|_{L^{2}\left(\Omega_{r}\right)}, \quad \forall q \in \mathbf{P}_{r}, \quad 1 \leq r \leq R,
$$

where $\gamma>0$ is independent of $h$. If there exists a subspace $\underset{\sim}{\overline{\mathbf{V}}_{h}}$ of $\underset{\sim}{\mathbf{V}_{h}}$ such that the pair $\left(\overline{\mathbf{V}}_{h}, \overline{\mathbf{P}}_{h}\right)$ satisfies (3.2), then $\left({\underset{\sim}{h}}_{h}, \mathbf{P}_{h}\right)$ also satisfies (3.2).

Proof. The proof is similar to the original one without weights $[6 ; 15$, p. 130]. Given $q \in \mathbf{P}_{h}$, we split it as follows:

$$
q=q^{*}+\bar{q},
$$

where

$$
\bar{q} \in \overline{\mathbf{P}}_{h},\left.\quad \bar{q}\right|_{\Omega_{r}}=\left|\Omega_{r}\right|^{-1} \int_{\Omega_{r}} q \quad \text { and }\left.\quad q^{*}\right|_{\Omega_{r}} \in \mathbf{P}_{r} .
$$

This is an orthogonal decomposition in $L^{2}\left(\Omega_{r}\right)$ for all $1 \leq r \leq R$. Hence,

$$
\|q\|_{L^{2}\left(\Omega_{r}\right)}^{2}=\left\|q^{*}\right\|_{L^{2}\left(\Omega_{r}\right)}^{2}+\|\bar{q}\|_{L^{2}\left(\Omega_{r}\right)}^{2} .
$$

The fact that $\operatorname{diam}\left(\Omega_{r}\right) \leq C h$, in conjunction with (2.2), yields

$$
C^{-1}\|q\|_{\sigma^{-2}, \Omega_{r}}^{2} \leq\left\|q^{*}\right\|_{\sigma^{-2}, \Omega_{r}}^{2}+\|\bar{q}\|_{\sigma^{-2}, \Omega_{r}}^{2} \leq C\|q\|_{\sigma^{-2}, \Omega_{r}}^{2},
$$

where $\|q\|_{\sigma^{-2}, \Omega_{r}}^{2}:=\int_{\Omega_{r}} q^{2} \sigma^{-2}$. Moreover, by (3.6) and (2.2), there exists $\underset{\sim}{v_{r}^{*}} \in$ $\mathbf{\sim}_{r}$ such that

$$
\begin{gathered}
\int_{\Omega_{r}} q^{*} \operatorname{div} \underset{\sim}{v_{r}^{*}}=\left\|q^{*}\right\|_{\sigma^{-2}, \Omega_{r}}^{2}, \\
\left\|\nabla{\underset{\sim}{v}}_{r}^{*}\right\|_{\sigma^{2}, \Omega_{r}} \leq C\left\|q^{*}\right\|_{\sigma^{-2}, \Omega_{r}} ;
\end{gathered}
$$

similarly, since $\left(\bar{\sim}_{h}, \overline{\mathbf{P}}_{h}\right)$ satisfies (3.2), there exists $\underset{\sim}{\bar{v}} \in \underset{\sim}{\overline{\mathbf{V}}_{h}}$ such that

$$
\begin{gathered}
\int_{\Omega} \bar{q} \operatorname{div} \bar{\sim}=\|\bar{q}\|_{\sigma^{-2}}^{2}, \\
\|\nabla \bar{v}\|_{\sigma^{2}} \leq C|\log \theta|^{1 / 2}\|\bar{q}\|_{\sigma^{-2}} .
\end{gathered}
$$


Let $\underset{\sim}{v}{ }^{*} \in{\underset{\sim}{\mathbf{V}}}_{h}$ be defined by $\left.{\underset{\sim}{v}}^{*}\right|_{\Omega_{r}}={\underset{\sim}{v}}_{r}^{*}$ for all $1 \leq r \leq R$. Next set

$$
\underset{\sim}{v}:=v_{\sim}^{*}+\alpha \bar{v}
$$

where $\alpha=K|\log \theta|^{-1}$ and $K>0$ is to be selected later on. From (3.11) it follows that

$$
\left\langle\operatorname{div} \underset{\sim}{\bar{v}}, q^{*}\right\rangle \leq C|\log \theta|^{1 / 2}\|\bar{q}\|_{\sigma^{-2}}\left\|q^{*}\right\|_{\sigma^{-2}} .
$$

Therefore, by (3.8) and (3.10), we have

$$
\begin{aligned}
\langle\operatorname{div} \underset{\sim}{v}, q\rangle & \geq\left\|q^{*}\right\|_{\sigma^{-2}}^{2}+\alpha\|\bar{q}\|_{\sigma^{-2}}^{2}-\alpha C|\log \theta|^{1 / 2}\|\bar{q}\|_{\sigma^{-2}}\left\|q^{*}\right\|_{\sigma^{-2}} \\
& \geq\left(1-\alpha \frac{C}{\varepsilon}|\log \theta|\right)\left\|q^{*}\right\|_{\sigma^{-2}}^{2}+\alpha(1-C \varepsilon)\|\bar{q}\|_{\sigma^{-2}}^{2} .
\end{aligned}
$$

A proper choice of $\varepsilon>0$ and $K$ implies

$$
\langle\operatorname{div} \underset{\sim}{v}, q\rangle \geq C\left(\left\|q^{*}\right\|_{\sigma^{-2}}^{2}+|\log \theta|^{-1}\|\bar{q}\|_{\sigma^{-2}}^{2}\right) .
$$

On the other hand, (3.9) and (3.11) together with the definition of $\alpha$ yield

$$
\|\nabla \underset{\sim}{v}\|_{\sigma^{2}} \leq C\left(\left\|q^{*}\right\|_{\sigma^{-2}}+|\log \theta|^{-1 / 2}\|\bar{q}\|_{\sigma^{-2}}\right),
$$

which concludes the proof of the lemma.

We now end this section with some applications of Lemma 3.3 to well-known finite element spaces. First, let $\Omega$ be partitioned into triangles.

Example 3.3. Taylor-Hood Element. This popular finite element is defined by $[15,20]$

$$
{\underset{\sim}{h}}_{\mathbf{V}_{T}}:=\left[P_{2}(T)\right]^{2},\left.\quad \mathbf{P}_{h}\right|_{T}:=P_{1}(T), \quad \forall T \in \mathscr{T}_{h} \quad \text { and } \quad \mathbf{P}_{h} \subset C^{0}(\Omega) ;
$$

thus $k=2$. The local condition (3.6) is valid [15, p. 178], whereas the global link is provided by $\left.\overline{\mathbf{V}}_{h}\right|_{T}=\left[P_{2}(T)\right]^{2}$ and $\left.\overline{\mathbf{P}}_{h}\right|_{T}=P_{0}(T)$ which verify (3.2) (see Example 3.2). A direct proof of (3.2) can be readily carried out by repeating the original one by Bercovier and Pironneau [4] and Verfürth [22], this time with weights.

Example 3.4. Crouzeix-Raviart Element. Let $\tilde{P}_{l}(T)$ be the space of homogeneous polynomials of degree $l$ restricted to $T$. Let $k \geq 2$ and set $[9,15]$

$$
\left.\underset{\sim}{\mathbf{V}_{h}}\right|_{T}:=\left[P_{k}(T) \oplus \lambda_{1} \lambda_{2} \lambda_{3} \tilde{P}_{k-2}(T)\right]^{2},\left.\quad \mathbf{P}_{h}\right|_{T}:=P_{k-1}(T), \quad \forall T \in \mathscr{T}_{h} .
$$

The local condition (3.6) follows easily from taking single elements to be macroelements $\Omega_{r}\left[15\right.$, p. 141]. The spaces $\overline{\mathbf{V}}_{h}$ and $\overline{\mathbf{P}}_{h}$ are as in Example 3.3.

We finally conclude with an example of quadrilateral elements which also satisfy (3.2). Let $\Omega$ be decomposed into convex quadrilaterals. 
Example 3.5. Let $k \geq 2$ and set

$$
\left.{\underset{\sim}{h}}_{h}\right|_{T}:=\left[Q_{k}(T)\right]^{2} \quad \text { and }\left.\quad \mathbf{P}_{h}\right|_{T}:=P_{k-1}(T), \quad \forall T \in \mathscr{T}_{h},
$$

where $Q_{k}$ stands for the space of polynomials of degree not greater than $k$ in each single variable. The inf-sup condition (3.2) is verified in a similar fashion to that above [15, p. 156].

\section{ERROR ANALYSIS}

The present goal is to prove that under the sole assumption (3.2), quasioptimal error estimates in the maximum norm follow for both velocity and pressure. This may be regarded as a natural extension of the standard theory in energy norm [7, 15]. Our main tool is the method of weighted Sobolev norms introduced by Natterer [17] and Nitsche [18].

An outline of the analysis is as follows. We first obtain an error estimate for the velocity gradient $\nabla \underset{\sim}{u}$ in terms of errors for the velocity $\underset{\sim}{u}$ and pressure $p$. We next derive an error bound for $u$ depending on the errors for $\nabla u$ and $p$. The weighted inf-sup condition finally provides an error estimate for $p$ as a function of the error bound for $\nabla u$, and allows the successful assembly of all partial results to produce rates of convergence for both physical variables. A similar strategy was previously used in [10].

Before we get started, we need some further notation, namely,

$$
{\underset{\sim}{e}}_{u}=\underset{\sim}{u}-{\underset{\sim}{u}}_{h}, \quad e_{p}=p-p_{h} ; \quad{\underset{\sim}{e}}_{u}=\underset{\sim}{\hat{u}}-{\underset{\sim}{u}}_{h}, \quad \hat{e}_{p}=\hat{p}-p_{h},
$$

where $\underset{\sim}{\hat{u}}$ and $\hat{p}$ stand for local interpolants of $\underset{\sim}{u}$ and $p$, respectively. We also set

$$
\begin{gathered}
E_{h}:=\|\nabla(\underset{\sim}{u}-\underset{\sim}{\hat{u}})\|_{\sigma^{-2}}^{2}+\|\underset{\sim}{u}-\underset{\sim}{\hat{u}}\|_{\sigma^{-4}}^{2}+\|p-\hat{p}\|_{\sigma^{-2}}^{2} ; \\
\theta:=K h|\log h|, \quad \eta:=(K|\log h|)^{-1},
\end{gathered}
$$

where $K>0$ is a large parameter to be selected. The error equations read as follows:

$$
\begin{aligned}
\left\langle\nabla \underset{\sim}{e_{u}}, \nabla \underset{\sim}{v}\right\rangle-\left\langle\operatorname{div} \underset{\sim}{v}, e_{p}\right\rangle=0, & \forall \underset{\sim}{v} \in{\underset{\sim}{\mathbf{V}}}_{h}, \\
\langle\operatorname{div} \underset{\sim}{\underset{\sim}{e}}, q\rangle=0, & \forall q \in \mathbf{P}_{h} .
\end{aligned}
$$

The quasi-optimal pointwise error estimates are summarized in the following theorem.

Theorem 4.1. There exists a constant $C>0$ independent of $h$ such that

$$
\begin{aligned}
& (h|\log h|)^{-1}\left\|{\underset{\sim}{e} u}_{u}\right\|_{L^{\infty}(\Omega)}+\left\|\nabla{\underset{\sim}{u}}_{u}\right\|_{L^{\infty}(\Omega)}+|\log h|^{-1 / 2}\left\|e_{p}\right\|_{L^{\infty}(\Omega)} \\
& \leq C|\log h|^{2}\left(\inf _{v \in \mathbf{V}_{h}}\|\nabla(\underset{\sim}{u}-\underset{\sim}{v})\|_{L^{\infty}(\Omega)}+\inf _{q \in \mathbf{P}_{h}}\|p-q\|_{L^{\infty}(\Omega)}\right),
\end{aligned}
$$

for all pairs $\left(\mathbf{V}_{h}, \mathbf{P}_{h}\right)$ satisfying the weighted inf-sup condition (3.2).

The proof of (4.3) will be split into three steps. Our first task is to demonstrate the following (partial) error estimate for the velocity gradient. 
Lemma 4.1. There exist constants $K_{0}, C>0$ such that

$$
\left\|\nabla \underset{\sim}{e_{u}}\right\|_{\sigma^{-2}}^{2} \leq C\left[\left\|\underset{\sim}{e_{u}}\right\|_{\sigma^{-4}}^{2}+\left(\frac{h^{2}}{\theta^{2}}+\eta\right)\left\|e_{p}\right\|_{\sigma^{-2}}^{2}+\eta^{-1} E_{h}\right],
$$

for all $\theta \geq K_{0} h$.

Proof. Note first that the following equality holds:

$$
\begin{aligned}
& \left\|\nabla \underset{\sim}{e_{u}}\right\|_{\sigma^{-2}}^{2}=\left\langle\nabla \underset{\sim}{e_{u}}, \sigma^{-2} \nabla(\underset{\sim}{u}-\hat{\sim})\right\rangle-\left\langle\nabla \underset{\sim}{e_{u}},{\underset{\sim}{\hat{e}_{u}}}_{u} \cdot \nabla \sigma^{-2}\right\rangle \\
& +\left\langle\nabla{\underset{\sim}{e}}_{u}, \nabla\left(\sigma^{-2} \hat{\sim}_{u}\right)\right\rangle=: \text { I }+ \text { II + III . }
\end{aligned}
$$

The first two terms can be easily handled in view of (2.3), namely,

$$
\mathrm{I}, \mathrm{II} \leq \varepsilon\left\|\nabla \underset{\sim}{e_{u}}\right\|_{\sigma^{-2}}^{2}+C \varepsilon^{-1}\left\|{\underset{\sim}{u}}_{u}\right\|_{\sigma^{-4}}^{2}+C \varepsilon^{-1} E_{h} .
$$

Hereafter, $\varepsilon>0$ will indicate a small parameter to be specified later on. Next set $\underset{\sim}{\psi}:=\sigma^{-2} \hat{\sim}_{u}$ and use (4.1) to rewrite the remaining term as follows:

$$
\mathrm{III}=\left\langle\nabla \underset{\sim}{e_{u}}, \nabla(\underset{\sim}{\psi}-\underset{\sim}{\hat{\psi}})\right\rangle+\left\langle\operatorname{div}(\underset{\sim}{\hat{\psi}}-\underset{\sim}{\psi}), e_{p}\right\rangle+\left\langle\operatorname{div} \underset{\sim}{\psi}, e_{p}\right\rangle=: \mathrm{IV}+\mathrm{V}+\mathrm{VI} .
$$

The first two terms are again easy to bound, now by virtue of (2.6). Indeed, we have

$$
\begin{aligned}
& \mathrm{IV}+\mathrm{V} \leq C \frac{h}{\theta}\left(\left\|\underset{\sim}{e_{u}}\right\|_{\sigma^{-2}}+\left\|e_{p}\right\|_{\sigma^{-2}}\right)\left(\left\|{\underset{\sim}{u}}_{u}\right\|_{\sigma^{-4}}+\left\|\nabla \underset{\sim}{\hat{e}_{u}}\right\|_{\sigma^{-2}}\right) \\
& \leq\left(\varepsilon+K_{0}^{-1}\right)\left\|\nabla{\underset{\sim}{u}}_{u}\right\|_{\sigma^{-2}}^{2} \\
& +C\left[\varepsilon^{-1} \frac{h^{2}}{\theta^{2}}\left\|e_{p}\right\|_{\sigma^{-2}}^{2}+\left\|e_{\sim}\right\|_{\sigma^{-4}}^{2}+\left(1+\varepsilon^{-1} \frac{h^{2}}{\theta^{2}}\right) E_{h}\right] .
\end{aligned}
$$

Making use of the definition of $\underset{\sim}{\psi}$, we obtain

$$
\left.\mathrm{VI}=\left\langle\operatorname{div} \underset{\sim}{\hat{\sim}_{u}}, \sigma^{-2} e_{p}\right\rangle-\left\langle(\underset{\sim}{u}-\underset{\sim}{\hat{u}}) \cdot \nabla \sigma^{-2}, e_{p}\right\rangle+\underset{\sim}{e_{u}} \cdot \nabla \sigma^{-2}, e_{p}\right\rangle=: \mathrm{VII}+\mathrm{VIII}+\mathrm{IX} .
$$

A simple calculation based on (4.2) and (2.5) gives

$$
\begin{aligned}
& \mathrm{VII}=\left\langle\operatorname{div} \underset{\sim}{\underset{\sim}{e}}, \sigma^{-2} \hat{e}_{p}-I_{h}\left(\sigma^{-2} \hat{e}_{p}\right)\right\rangle-\left\langle\operatorname{div}(\underset{\sim}{u}-\underset{\sim}{\hat{u}}), \sigma^{-2} e_{p}\right\rangle+\left\langle\operatorname{div} \underset{\sim}{e_{u}}, \sigma^{-2}(p-\hat{p})\right\rangle \\
& \leq \varepsilon\left\|\nabla e_{\sim}\right\|_{\sigma^{-2}}^{2}+C \varepsilon^{-1}\left(\frac{h^{2}}{\theta^{2}}+\eta\right)\left\|e_{p}\right\|_{\sigma^{-2}}^{2}+C \varepsilon^{-1}\left(\frac{h^{2}}{\theta^{2}}+\eta^{-1}\right) E_{h} .
\end{aligned}
$$

At the same time, (2.3) implies

$$
\text { VIII } \leq C\|\underset{\sim}{u}-\hat{\sim}\|_{\sigma^{-4}}\left\|e_{p}\right\|_{\sigma^{-2}} \leq C \eta\left\|e_{p}\right\|_{\sigma^{-2}}^{2}+C \eta^{-1} E_{h} .
$$

Term IX will be handled by means of a duality argument. Set $g:={\underset{\sim}{u}}_{u} \cdot \nabla \sigma^{-2} \in$ $H_{0}^{1}(\Omega)$ and let $(\underset{\sim}{v}, q) \in \underset{\sim}{\mathbf{V}} \times \mathbf{P}$ be the solution to the auxiliary problem

$$
\begin{aligned}
-\Delta v+\nabla q & =\underset{\sim}{0}, \\
\operatorname{div} v & =g-m \phi,
\end{aligned}
$$


which, by Lemma 2.3 and (2.1), satisfies

$$
\left\|D_{\sim}^{2} \underset{\sim}{v}\right\|_{\sigma^{2}}+\|\nabla q\|_{\sigma^{2}} \leq C \theta^{-1}\left(\left\|\nabla \underset{\sim}{e_{u}}\right\|_{\sigma^{-2}}+\left\|{\underset{\sim}{u}}_{u}\right\|_{\sigma^{-4}}\right) .
$$

Hence,

$$
\mathrm{IX}=\left\langle\operatorname{div}(\underset{\sim}{v}-\underset{\sim}{v}), e_{p}\right\rangle+\left\langle\operatorname{div} \underset{\sim}{\hat{v}}, e_{p}\right\rangle+m\left\langle\phi, e_{p}\right\rangle=: \mathrm{X}+\mathrm{XI}+\mathrm{XII},
$$

and we now examine these three terms separately. By (4.7), we easily get

$$
\mathrm{X} \leq C h\left\|e_{p}\right\|_{\sigma^{-2}}\left\|D_{\sim}^{2} \underset{\sim}{v}\right\|_{\sigma^{2}} \leq \varepsilon\left\|\nabla \underset{\sim}{e_{u}}\right\|_{\sigma^{-2}}^{2}+\left\|{\underset{\sim}{u}}_{u}\right\|_{\sigma^{-4}}^{2}+C \varepsilon^{-1} \frac{h^{2}}{\theta^{2}}\left\|e_{p}\right\|_{\sigma^{-2}}^{2} .
$$

Making use of (4.1),(4.2) and (4.6), we further split XI as follows:

$$
\mathrm{XI}=\left\langle\nabla \underset{\sim}{e_{u}}, \nabla(\underset{\sim}{\hat{v}}-\underset{\sim}{v})\right\rangle+\langle\operatorname{div} \underset{\sim}{\underset{\sim}{e}}, q-\hat{q}\rangle
$$

thus

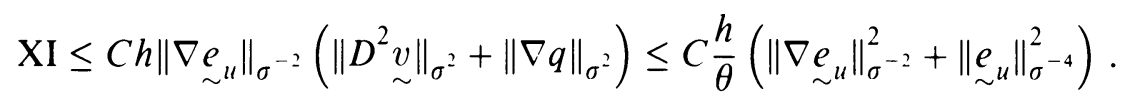

By virtue of (4.2), (2.3) and (2.4), we deduce that

$$
\begin{aligned}
m & =\left\langle\underset{\sim}{\underset{\sim}{e}}, \nabla \sigma^{-2}\right\rangle=\left\langle\operatorname{div} \underset{\sim}{\underset{\sim}{e}}, I_{h}\left(\sigma^{-2}\right)-\sigma^{-2}\right\rangle \\
& \leq C h\|\nabla \underset{\sim}{\underset{\sim}{e}}\|_{\sigma^{-2}}\left\|\nabla \sigma^{-2}\right\|_{\sigma^{2}} \leq C \frac{h}{\theta}\|\nabla \underset{\sim}{\underset{\sim}{e}}\|_{\sigma^{-2}},
\end{aligned}
$$

and, as a result, that

$$
\mathrm{XII} \leq C \frac{h}{\theta}\left\|\nabla \underset{\sim}{e_{u}}\right\|_{\sigma^{-2}}\left\|e_{p}\right\|_{\sigma^{-2}} \leq \varepsilon\left\|\nabla \underset{\sim}{e_{u}}\right\|_{\sigma^{-2}}^{2}+C \varepsilon^{-1} \frac{h^{2}}{\theta^{2}}\left\|e_{p}\right\|_{\sigma^{-2}}^{2} .
$$

Collecting all above estimates and inserting them in (4.5), we realize that a suitable choice of $\varepsilon$ and $K_{0}$ allows the term $\left\|\nabla e_{\sim l}\right\|_{\sigma^{-2}}^{2}$ appearing in the righthand side of the resulting expression to be absorbed into its left side, thus yielding the desired estimate (4.4).

Remark 4.1. Instead of the duality argument (4.6), we may have applied the obvious inequality

$$
\mathrm{IX} \leq C \eta\left\|e_{p}\right\|_{\sigma^{-2}}^{2}+C \eta^{-1}\left\|e_{\sim}\right\|_{\sigma^{-4}}^{2} .
$$

This would have led, however, to higher powers of logarithmic factors in the final estimates.

Remark 4.2. Let $\underset{\sim}{\hat{u}}=\Pi_{h} \underset{\sim}{u}$, where $\Pi_{h}$ satisfies (3.3) and is defined locally; thus $\Pi_{h}$ is an optimal interpolation operator in weighted norms. Then we split term VI above as follows:

$$
\mathrm{VI}=\left\langle\operatorname{div} \hat{\sim}_{u}, \sigma^{-2} e_{p}\right\rangle+\left\langle\underset{\sim}{\hat{e}_{u}} \cdot \nabla \sigma^{-2}, e_{p}\right\rangle=: A+B .
$$

In evaluating term $A$ we make use of the required properties on $\Pi_{h}$, together with (2.5) and (4.2), to arrive at

$$
\begin{aligned}
A & =\left\langle\operatorname{div}{\underset{\sim}{\hat{e}_{u}}}_{1}, \sigma^{-2} \hat{e}_{p}-I_{h}\left(\sigma^{-2} \hat{e}_{p}\right)\right\rangle+\left\langle\operatorname{div} \underset{\sim}{\hat{e}_{u}}, \sigma^{-2}(p-\hat{p})\right\rangle \\
& \leq \varepsilon\left\|\nabla{\underset{\sim}{u}}_{u}\right\|_{\sigma^{-2}}^{2}+C \varepsilon^{-1} \frac{h^{2}}{\theta^{2}}\left\|e_{p}\right\|_{\sigma^{-2}}^{2}+C \varepsilon^{-1} E_{h} .
\end{aligned}
$$


The analysis of term $B$ is similar to that of term IX above. In fact, we exploit the local character of $\Pi_{h}$ to replace $\underset{\sim}{\hat{e}_{u}}$ by $\underset{\sim}{e_{u}}$ in (4.7) and next deal with $m$ as follows:

$$
m=\left\langle\operatorname{div}{\underset{\sim}{e}}_{u}, I_{h}\left(\sigma^{-2}\right)-\sigma^{-2}\right\rangle \leq C \frac{h}{\theta}\left\|\nabla{\underset{\sim}{e}}_{u}\right\|_{\sigma^{-2}} .
$$

These estimates lead to the following slight improvement of (4.4):

$$
\left\|\nabla \underset{\sim}{e_{u}}\right\|_{\sigma^{-2}}^{2} \leq C\left(\left\|{\underset{\sim}{u} u}_{\sigma^{-4}}\right\|_{\sigma^{-4}}+\frac{h^{2}}{\theta^{2}}\left\|e_{p}\right\|_{\sigma^{-2}}^{2}+E_{h}\right) \text {. }
$$

The second step in our error analysis consists of deriving a (partial) error bound for the velocity.

Lemma 4.2. There exists a constant $C>0$ such that

$$
\left\|e_{\sim}\right\|_{\sigma^{-4}}^{2} \leq C \frac{h^{2}}{\theta^{2}}|\log \theta|\left(\left\|\nabla e_{\sim}\right\|_{\sigma^{-2}}^{2}+\left\|e_{p}\right\|_{\sigma^{-2}}^{2}\right) \text {. }
$$

Proof. We employ a duality argument. Let $\underset{\sim}{v}, q) \in \underset{\sim}{\mathbf{V}} \times \mathbf{P}$ be the solution to the auxiliary problem

$$
\begin{gathered}
-\Delta \underset{\sim}{v}+\nabla q=\sigma^{-4} \underset{\sim}{e_{u},} \\
\operatorname{div} \underset{\sim}{v}=0,
\end{gathered}
$$

which, in view of (2.13), satisfies

$$
\left\|D^{2} \underset{\sim}{v}\right\|_{\sigma^{2}}+\|\nabla q\|_{\sigma^{2}} \leq C \theta^{-1}|\log \theta|^{1 / 2}\left\|{\underset{\sim}{u}}_{u}\right\|_{\sigma^{-4}}
$$

Consequently, making use of (4.1), (4.2) and (4.9), we can express $\left\|\underset{\sim}{e_{u}}\right\|_{\sigma^{-4}}$ as follows:

$$
\left\|{\underset{\sim}{e} u}_{u}\right\|_{\sigma^{-4}}^{2}=\left\langle\nabla \underset{\sim}{e_{u}}, \nabla(\underset{\sim}{v}-\underset{\sim}{\hat{v}})\right\rangle+\left\langle\operatorname{div}(\underset{\sim}{\hat{v}}-\underset{\sim}{v}), e_{p}\right\rangle+\left\langle\operatorname{div} \underset{\sim}{e_{u}}, \hat{q}-q\right\rangle .
$$

By (4.10), we thus have

$$
\begin{aligned}
\left\|\underset{\sim}{e_{u}}\right\|_{\sigma^{-4}}^{2} & \leq C h\left(\left\|\nabla \underset{\sim}{e_{u}}\right\|_{\sigma^{-2}}+\left\|e_{p}\right\|_{\sigma^{-2}}\right)\left(\left\|D_{\underset{\sim}{2}}^{2}\right\|_{\sigma^{2}}+\|\nabla q\|_{\sigma^{2}}\right) \\
& \leq C \frac{h}{\theta}|\log \theta|^{1 / 2}\left(\left\|\nabla \underset{\sim}{{\underset{\sim}{u}}_{u}}\right\|_{\sigma^{-2}}+\left\|e_{p}\right\|_{\sigma^{-2}}\right)\left\|\underset{\sim}{e_{u}}\right\|_{\sigma^{-4}},
\end{aligned}
$$

which clearly implies the assertion (4.8).

Remark 4.3. Let $\underset{\sim}{\hat{v}}=\Pi_{h} \underset{\sim}{v}$, where $\Pi_{h}$ is a local interpolation operator which verifies (3.3). Then, the middle term in the above expression of $\left\|e_{\sim}\right\|_{\sigma^{-4}}^{2}$ can be also handled as follows:

$$
\left\langle\operatorname{div}(\underset{\sim}{\hat{v}}-\underset{\sim}{v}), e_{p}\right\rangle=\langle\operatorname{div}(\underset{\sim}{\hat{v}}-\underset{\sim}{v}), p-\hat{p}\rangle \leq C \frac{h}{\theta}|\log \theta|^{1 / 2}\|\underset{\sim}{\underset{\sim}{e} u}\|_{\sigma^{-4}}\|p-\hat{p}\|_{\sigma^{-2}} .
$$

This results in the following substitute for (4.8):

$$
\left\|{\underset{\sim}{e} u}_{u}\right\|_{\sigma^{-4}}^{2} \leq C \frac{h^{2}}{\theta^{2}}|\log \theta|\left(\left\|\nabla \underset{\sim}{e_{u}}\right\|_{\sigma^{-2}}^{2}+E_{h}\right) .
$$

The final step entails the use of the weighted inf-sup condition to produce a (partial) error bound for the pressure. 
Lemma 4.3. There exists a constant $C>0$ such that

$$
\left\|e_{p}\right\|_{\sigma^{-2}}^{2} \leq C|\log \theta|\left(\left\|\nabla e_{\sim}\right\|_{\sigma^{-2}}^{2}+E_{h}\right) \text {. }
$$

Proof. Set $q:=\hat{p}-p_{h}-|\Omega|^{-1} \int_{\Omega} \hat{p} \in \mathbf{P}_{h}$. Then, for all $\underset{\sim}{v} \in \underset{\sim}{\mathbf{V}_{h}}$, we have

$$
\begin{aligned}
\langle\operatorname{div} \underset{\sim}{v}, q\rangle & =\left\langle\operatorname{div} \underset{\sim}{v}, e_{p}\right\rangle-\langle\operatorname{div} \underset{\sim}{v}, p-\hat{p}\rangle-\left\langle\operatorname{div} \underset{\sim}{v},|\Omega|^{-1} \int_{\Omega} \hat{p}\right\rangle \\
& =\langle\nabla \underset{\sim}{v}, \underset{\sim}{\underset{f}{e}}\rangle-\langle\operatorname{div} \underset{\sim}{v}, p-\hat{p}\rangle \\
& \leq\|\nabla \underset{\sim}{v}\|_{\sigma^{2}}\left(\left\|\nabla{\underset{\sim}{e} u}_{u}\right\|_{\sigma^{-2}}+E_{h}\right),
\end{aligned}
$$

as a consequence of (4.1) and the fact that the rightmost term in the first line vanishes. Using now the weighted inf-sup condition (3.2) yields

$$
\|q\|_{\sigma^{-2}} \leq C|\log \theta|^{1 / 2} \sup _{\substack{v \in \mathfrak{V}_{h} \\ \sim}} \frac{\langle\operatorname{div} \underset{\sim}{v}, q\rangle}{\|\nabla \underset{\sim}{v}\|_{\sigma^{2}}} \leq C|\log \theta|^{1 / 2}\left(\left\|\nabla \underset{\sim}{e_{u}}\right\|_{\sigma^{-2}}+E_{h}\right) .
$$

Finally, since $p$ has mean value zero, we arrive at

$$
\begin{aligned}
\left\|e_{p}\right\|_{\sigma^{-2}} & \leq\|p-\hat{p}\|_{\sigma^{-2}}+\|q\|_{\sigma^{-2}}+\left\||\Omega|^{-1} \int_{\Omega}(p-\hat{p})\right\|_{\sigma^{-2}} \\
& \leq C|\log \theta|^{1 / 2}\left(\left\|\nabla \underset{\sim}{e_{u}}\right\|_{\sigma^{-2}}+E_{h}\right),
\end{aligned}
$$

concluding the proof of the lemma.

What remains to be done is to assemble the partial results $(4.4),(4.8)$ and (4.11) to end up with global error estimates in weighted Sobolev norms. We first obtain

$$
\begin{aligned}
\left\|\nabla \underset{\sim}{e_{u}}\right\|_{\sigma^{-2}}^{2} & \leq C\left(\frac{h^{2}}{\theta^{2}}|\log \theta|^{2}+\eta|\log \theta|\right)\left(\|\nabla \underset{\sim}{\underset{\sim}{e}}\|_{\sigma^{-2}}^{2}+E_{h}\right)+C \eta^{-1} E_{h} \\
& \leq C K^{-1}\left\|\nabla \underset{\sim}{e_{u}}\right\|_{\sigma^{-2}}^{2}+C\left(K^{-1}+K|\log h|\right) E_{h},
\end{aligned}
$$

where we have used the definitions of both $\theta$ and $\eta$. Then, taking $K$ sufficiently large leads to the following bound for $\nabla \underset{\sim}{e_{u}}$ :

$\left\|\nabla{\underset{\sim}{u}}_{u}\right\|_{\sigma^{-2}}^{2} \leq C|\log h| E_{h}=C|\log h|\left(\|\nabla(\underset{\sim}{\mathcal{u}}-\underset{\sim}{\hat{u}})\|_{\sigma^{-2}}^{2}+\|\underset{\sim}{\mathcal{u}}-\underset{\sim}{\hat{u}}\|_{\sigma^{-4}}^{2}+\|p-\hat{p}\|_{\sigma^{-2}}^{2}\right)$.

In order to derive error bounds for velocity and pressure, we just have to replace the latter estimate in (4.8) and (4.11), respectively. We then find

$$
\left\|{\underset{\sim}{u}}_{u}\right\|_{\sigma^{-4}}^{2} \leq C \frac{h^{2}}{\theta^{2}}|\log \theta|^{3} E_{h}, \quad\left\|e_{p}\right\|_{\sigma^{-2}}^{2} \leq C|\log \theta|^{2} E_{h} .
$$

We are now in a position to prove Theorem 4.1. Let $x_{0}$ in (2.1) be chosen so that $\left|\nabla \underset{\sim}{\hat{e}_{u}}\left(x_{0}\right)\right|=\left\|\nabla{\underset{\sim}{\hat{e}}}_{u}\right\|_{L^{\infty}(\Omega)}$. The fact that $\nabla{\underset{\sim}{\hat{e}}}_{u}$ is piecewise polynomial, 
coupled with (2.4), yields

$$
\begin{aligned}
& \left\|\nabla{\underset{\sim}{u}}_{u}\right\|_{L^{\infty}(\Omega)} \leq\left\|\nabla \underset{\sim}{\hat{e}_{u}}\right\|_{L^{\infty}(\Omega)}+\|\nabla(\underset{\sim}{u}-\underset{\sim}{\hat{u}})\|_{L^{\infty}(\Omega)} \\
& \leq C \frac{\theta}{h}\|\nabla \underset{\sim}{\hat{e}}\|_{\sigma^{-2}}+\|\nabla(\underset{\sim}{u}-\underset{\sim}{\hat{u}})\|_{L^{\infty}(\Omega)} \\
& \leq C \frac{\theta}{h}|\log h|^{1 / 2} E_{h}^{1 / 2}+\|\nabla(\underset{\sim}{u}-\underset{\sim}{\hat{u}})\|_{L^{\infty}(\Omega)} \\
& \leq C|\log h|^{2}\left(\|\nabla(\underset{\sim}{u}-\underset{\sim}{\hat{u}})\|_{L^{\infty}(\Omega)}+\|p-\hat{p}\|_{L^{\infty}(\Omega)}\right) .
\end{aligned}
$$

A similar calculation, now with a different choice of $x_{0}$, leads to the remaining estimates for velocity and pressure. The theorem is thus proved.

Remark 4.4. The quasi-optimal pointwise error estimates in (4.3) contain logarithmic factors which are probably not sharp. The main reason why they are worse than those for the Poisson's equation, produced by the method of weighted Sobolev norms, is the logarithm appearing in the weighted inf-sup condition (3.2). However, we may improve upon (4.3) whenever the operator $\Pi_{h}$ in (3.3), (3.4) can be constructed locally. In fact, combining Remarks 4.2 and 4.3 with Lemma 4.3 , and choosing $\theta=K h|\log h|^{1 / 2}$, we find

$$
\begin{aligned}
& h^{-1}\left\|{\underset{\sim}{e} u}_{L^{\prime}(\Omega)}+|\log h|^{1 / 2}\right\| \nabla \underset{\sim}{e_{u}}\left\|_{L^{\infty}(\Omega)}+\right\| e_{p} \|_{L^{\infty}(\Omega)} \\
& \leq C|\log h|^{3 / 2}\left(\inf _{\substack{v \in \mathbf{V}_{h} \\
\sim}}\|\nabla(\underset{\sim}{u}-\underset{\sim}{v})\|_{L^{\infty}(\Omega)}+\inf _{q \in \mathbf{P}_{h}}\|p-q\|_{L^{\infty}(\Omega)}\right) .
\end{aligned}
$$

This result is similar to that in [10] but is still a bit worse than the one in [11].

Remark 4.5. It would be of interest to know whether the logarithms could be completely removed for $k$ (degree of interpolation polynomials) $>1$, as happens for the Poisson's equation.

\section{BIBLIOGRAPHY}

1. D. N. Arnold, F. Brezzi and M. Fortin, A stable finite element for the Stokes equations, Calcolo 21 (1984), 337-344.

2. D. N. Arnold, L. R. Scott and M. Vogelius, Regular inversion of the divergence operator with Dirichlet boundary conditions on a polygon, Ann. Scuola Norm. Sup. Pisa 15 (1988), 169-192.

3. I. Babuška, Error bounds for the finite element method, Numer. Math. 16 (1971), 322-333.

4. M. Bercovier and O. Pironneau, Error estimates for finite element method solution of the Stokes problem in the primitive variables, Numer. Math. 33 (1979), 211-224.

5. C. Bernardi and B. Raugel, Analysis of some finite elements for the Stokes problem, Math. Comp. 44 (1985), 71-79.

6. J. M. Boland and R. A. Nicolaides, Stability of some elements under divergence constraint, SIAM J. Numer. Anal. 20 (1983), 722-731.

7. F. Brezzi, On the existence, uniqueness and approximation of saddle-point problems arising from Lagrangian multipliers, RAIRO Anal. Numér. 8 (1974), 129-151.

8. P. G. Ciarlet, The finite element method for elliptic problems, North-Holland, Amsterdam, 1978.

9. M. Crouzeix and P.-A. Raviart, Conforming and nonconforming finite element methods for solving the stationary Stokes equations I, RAIRO Anal. Numér. 7, R-3 (1973), 33-75. 
10. R. G. Durán and R. H. Nochetto, Pointwise accuracy of a Petrov-Galerkin approximation to the Stokes problem, SIAM J. Numer. Anal. 26 (1989) (to appear).

11. R. G. Durán, R. H. Nochetto and J. Wang, Sharp maximum norm error estimates for finite element approximations of the Stokes problem in 2-D, Math. Comp. 51 (1988), 491-506.

12. R. S. Falk and J. E. Osborn, Error estimates for mixed methods, RAIRO Anal. Numér. 14 (1980), 249-277.

13. M. Fortin, An analysis of the convergence of mixed finite element methods, RAIRO Anal. Numér. 11 (1977), 341-354.

14. L. Gastaldi and R. H. Nochetto, Sharp maximum norm error estimates for general mixed finite element approximations to second order elliptic equations, RAIRO Modél. Math. Anal. Numér. 23 (1989), 103-128.

15. V. Girault and P. A. Raviart, Finite element methods for Navier-Stokes equations: theory and applications, Springer-Verlag, Berlin and Heidelberg, 1986.

16. R. B. Kellogg and J. E. Osborn, A regularity result for the Stokes problem in a convex polygon, J. Funct. Anal. 21 (1976), 397-431.

17. F. Natterer, Über die punktweise Konvergenz finiter Elemente, Numer. Math. 25 (1975), 67-78.

18. J. A. Nitsche, $L^{\infty}$-convergence of finite element approximations, Mathematical Aspects of the Finite Element Method, Lecture Notes in Math., vol. 606, Springer-Verlag, New York, 1977, pp. 261-274.

19. G. Talenti, Best constant in Sobolev inequality, Ann. Mat. Pura Appl. 110 (1976), 353-372.

20. C. Taylor and P. Hood, A numerical solution of the Navier-Stokes equations using the finite element method, Comput. \& Fluids 1 (1973), 73-100.

21. R. Temam, Navier-Stokes equations, North Holland, Amsterdam, 1984.

22. R. Verfürth, Error estimates for a mixed finite element approximation of the Stokes equations, RAIRO Anal. Numér. 18 (1984), 175-182.

Departamento de Matemática, Universidad de la Plata, P.O. Box 172, 1900 La Plata, ARGENTINA

Department of Mathematics and Institute for Physical Science and Technology, University of Maryland, College Park, Maryland 20742. E-mail: rhn@athena.umd.edu 